\title{
Actitudes ante prácticas inclusivas en Educación
}

\author{
Aura Hilda Rayo Pérez ${ }^{1}$ \\ Jilma Pereyra López ${ }^{2}$
}

\section{RESUMEN}

El presente artículo resume una investigación de carácter cualitativa, con enfoque fenomenológico realizada con la comunidad educativa en el centro escolar Monseñor "Oscar Arnulfo Romero" de la ciudad de Estelí, en el I semestre del año 2014. Con esta investigación se pretende conocer las actitudes de la comunidad educativa ante prácticas de la inclusividad educativa. Las técnicas aplicadas para la recolección de información fueron: entrevista a profundidad, encuestas, grupo focal y la escala tipo Likert para medir actitudes. La información obtenida con las técnicas aplicadas fue analizada en función de los objetivos formulados. Los resultados más relevantes indican que las actitudes ante prácticas inclusivas en este centro son inclusivas. Existe falta de estrategias metodológicas por parte de las maestras para atender a niños y niñas con necesidades educativas. Se da poca atención por técnicos metodológicos del Ministerio de Educación. Poca participación de padres-madres de familia en las actividades del centro escolar en función de contribuir a la formación y desarrollo de habilidades que preparan al niño-niña para la sociedad, independientemente de las características propias.

Palabras clave: Actitud, Necesidad Educativa, Inclusividad Educativa, Diversidad.

Recibido: 10 de diciembre de 2014

Aceptado: 10 de marzo de 2015

\footnotetext{
1 Egresada de la Maestría en Gestión del Desarrollo Comunitario, modalidad virtual Atención integral a la niñez. Gestiones en función del desarrollo comunitario. Correo Electrónico: arayo_24@yahoo.es

2 Maestra en Psicología, atención personalizada y Desarrollo Social/Especialista en Gestión del Desarrollo Comunitario /Psicóloga/ Docente en FAREM Estelí, UNAN Managua/Consultora independiente. Correo Electrónico: jplo42@, yahoo.com
} 


\title{
Attitudes toward inclusive practices in Education
}

\begin{abstract}
This article summarizes a research of qualitative character, with phenomenological approach performed with the educational community in the school Monsignor "Oscar Arnulfo Romero" in the city of Esteli, in the first half of 2014. This research aims to determine the attitudes of the educational community to practices of educational inclusiveness. The techniques used for data collection were: depth interviews, surveys, focus group and Likert scale to measure attitudes. The information obtained from the applied techniques was analyzed in terms of the stated objectives. The most relevant results indicate that attitudes to inclusive practices in this center are inclusive. There is a lack of methodological strategies by teachers to serve children with special educational needs. Little attention is given by methodological experts from the Ministry of Education. Low participation of fathers-mothers in the activities of the school in terms of contributing to the training and development of skills that prepares the boy-girl for society, regardless of characteristics.
\end{abstract}

Keywords: Attitude, Educational Need, Inclusivity Education, Diversity. 


\section{INTRODUCCIÓN}

Ainscow, Booth y Dyson, (2006), hacen referencia al respecto: "La educación inclusiva tiene el objetivo de asegurar el derecho a la educación de todos/as, cualesquiera que sean sus condiciones particularesindividuales".

Para que las escuelas sean inclusivas, es decir que las escuelas sean para todos/as, es imprescindible que el sistema educativo asegure que todos/as los/as estudiantes, tengan acceso a un aprendizaje significativo en los salones de clases.

Las razones que sustentan este hecho se consideran en los siguientes aspectos: El concepto de inclusión, manifiesta más claramente y con mayor exactitud, que todos/as los/as estudiantes deben estar incluidos en la vida educativa y social.

A la educación inclusiva se le acredita un concepto muy amplio que no está referido solamente a lograr el acceso a las escuelas regulares de determinados grupos tradicionalmente excluidos, sino que también tiene como finalidad, transformar el sistema educativo en su conjunto, para atender la diversidad de necesidades educativas que presentan los/as estudiantes, a fin de asegurar la igualdad de oportunidades y su plena participación.

La educación inclusiva implica eliminar las barreras que existan para el aprendizaje y abrir puertas a los/as estudiantes sin discriminación alguna. Maximizar los recursos que apoyen ambos procesos (inclusividad y educación), es trabajo arduo y constante con la finalidad de que las diferencias culturales, socioeconómicas, individuales y de género, no se conviertan en desigualdades educativas y por ende desigualdades sociales.

Las barreras se pueden encontrar en todos los aspectos y estructuras del sistema, ya sea en los centros educativos, en la comunidad o en las políticas locales y nacionales. Estas no solamente pueden impedir el acceso de los estudiantes al centro educativo, sino también limitar su aprendizaje, participación y desarrollo como individuo con igualdades en derechos.

El tema de la inclusividad educativa es muy sensible ante la sociedad, fue este el motivo de seleccionar el tema, dado que es política del gobierno y autoridades del Ministerio de Educación la atención a la niñez sin discriminación y hay centros educativos que no ponen en práctica estas políticas con técnicas y herramientas adecuadas.

\section{MATERIALES Y MÉTODOS}

Para fines del estudio se seleccionó personal de dirección, maestras de aula, padres-madres de familia, niños y niñas con y sin necesidades educativas del centro escolar monseñor "Oscar Arnulfo Romero". La muestra estuvo conformada por directora, subdirectora y consejera escolar, técnica metodológica del Ministerio de Educación, tres padres y dos madres de familia. Con el propósito de enriquecer los resultados de la investigación, también se aplicó la técnica tipo Likert para medir actitudes, se aplicó a la directora, subdirectora y consejera escolar, a una técnica del Ministerio de Educación, a catorce maestras de aula, nueve madres y a un padre de familia. El proceso de selección de las y los participantes fue de tipo no probabilístico.

Para la recolección de la información se aplicaron las técnicas de la entrevista a profundidad y grupo focal. La entrevista se basó en la elaboración de una guía de preguntas dirigidas con el objetivo de recopilar información de fuentes primarias maestras, padresmadres de familia. El grupo focal se realizó con quince niños y niñas del primer grado "A" (diez niñas $\mathrm{y}$ cinco niños) del turno matutino del centro escolar. 
El procesamiento de datos consistió en la revisión de resultados de las entrevistas a sujetos involucrados y la información obtenida a través del grupo focal con niños y niñas. El análisis e interpretación de datos se realizó en base a un sistema de categorías de análisis previamente establecidas de acuerdo a los objetivos propuestos. Con el propósito de garantizar confiabilidad al estudio se realizó la triangulación de información obtenida de las diferentes técnicas aplicadas. En este sentido se iba realizando un análisis comparativo del sentir, pensar y actuar de los sujetos involucrados.

Los resultados se organizaron de acuerdo a los objetivos propuestos en base a caracterizar, describir y explorar actitudes ante la inclusividad educativa del centro escolar monseñor "Oscar Arnulfo Romero".

\section{RESULTADOS}

En cuanto a las prácticas del centro escolar monseñor "Oscar Arnulfo Romero", los padres-madres de familia de niños y niñas con Necesidades Educativas asociadas o no a una discapacidad, afirman que la atención brindada por las docentes es de calidad y están satisfechos/as con la oportunidad que el centro les brinda al poder tener a su hijo/a en este centro.

Padres y madres de familia expresaron que: "La escuela brinda una atención excelente, por eso tengo a mi hijo/a aquí". "La maestra se ha encargado de atender a mi hijo y ha tenidos cambios positivos". "La conducta de mi hija ha mejorado desde que está en este centro escolar".

Otros/as padres/madres afirmaron: "Es triste ver tantos niños/as con Necesidades Educativas, yo quisiera que todos/as fueran "normales".

Lo expuesto anteriormente indica desacuerdo con la inclusividad, sin embargo se muestran contentos de los valores colaborativos que están aprendiendo sus hijos/as y lo expresan de la siguiente manera: "Me gusta que mi hija colabore ayudando a estos/as niños/ as que lo necesitan, por ejemplo a los que vienen en sillas de ruedas"

En cuanto a aspectos de la esfera afectiva por parte de las maestras se logró identificar satisfacción con las prácticas de inclusividad en el centro, cuando las y los padres-madres de familia expresan su confianza en la atención que el centro ofrece.

\section{DISCUSIÓN}

No se observó consejerías a niños y niñas ni atención psicológica, sin embargo la consejera escolar ha asistido a diplomados orientados por el MINED que han contribuido a la capacitación de este recurso sensible en la aplicación de las políticas inclusivas de este centro escolar.

Es evidente la preocupación del personal del centro por brindar una atención ordenada, de acuerdo a las necesidades de sus estudiantes, pero no es posible atender de manera individualizada cada caso. Conversar con los padres y madres de familia no es nada fácil, no demuestran interés por reforzar los aprendizajes de su hijo/a, esto atribuido al factor tiempo.

Se añaden las actividades lúdicas como una práctica del centro, pero en una esfera muy pobre, la actividad lúdica no solo es que la niñez tenga un espacio para realizar juegos libres, es importante la realización de juegos orientados por la facilitadora con el objetivo de desarrollar en el estudiante habilidades y destrezas que lo preparan para la vida.

\section{CONCLUSIONES}

Se tomó en cuenta el aporte de Ávila Durán y Esquivel Cordero (2009) en relación a que las prácticas en el marco de una Educación Inclusiva deben eliminar o minimizar barreras que existen y que impiden 
que todos/as los/as estudiantes puedan acceder al aprendizaje y lograr una plena participación en la escuela, sin diferencia de género, cultura, físicas, socioeconómicas, o de cualquier otra índole, estos no deben convertirse en factor de desigualdad educativa o en exclusión social".

A continuación se describen prácticas observadas en este centro escolar que minimizan o eliminan barreras para acceder a una educación inclusiva.

Matricula abierta para todo/a el que desea ingresar a este centro de estudios, atención individualizada para quien lo/la necesite, incluyendo niños-as con NE, infraestructura adecuada, de acuerdo a las necesidades de los estudiantes, participación y recreación. Se atiende a la niñez con dificultades de movilidad en la primera planta del centro escolar, en el horario de ingreso al centro, el personal se dispone a atender de manera adecuada a cada uno/a de los/as niños/ as asegurando el acceso hasta el salón de clases, el sistema de relaciones laborales es efectivo, se observa un ambiente de confianza y colaboración entre dirección - consejería - maestras y estudiantes, la dirección y personal docente practica la flexibilidad con los/as niños/as, con NE, tomando en cuenta sus características propias, planes evaluativos sistemáticos de los progresos de los estudiantes con NE.

En este centro se pone en práctica la inclusividad en función de desarrollar en los/as estudiantes hábitos de colaboración, participación, solidaridad con los/as compañeros/as, siendo esta una práctica que lleva al crecimiento de la formación en valores y académicos de la niñez y a incorporar hábitos de inclusión, para que sean integrados y vistos de la misma forma quienes presentan NE y quienes no las presentan. Estas prácticas de colaboración como son convivencia, integración, participación fortalecen principios de igualdad.

Los valores expresados en el quehacer educativo que se observaron se describen a continuación:

Se trabaja en el marco del respeto, compañerismo, solidaridad. El personal docente de la escuela considera que la diversidad es una forma de enriquecer conocimientos cada día y lleva a buscar nuevas formas de trabajo que facilitan el aprendizaje, las maestras comparten sobre problemáticas encontradas y juntas construyen alternativas de solución, generalmente lo hacen mediante talleres, círculos de calidad, cursos de estrategias de educación inclusiva, pero el trabajo no se les facilita por razones diversas; no disponen de tiempo para organizar actividades de acuerdo a necesidades individuales.

\section{BIBLIOGRAFÍA}

Aiscow, B. (2006). El Dilema en la Educación Inclusiva. Reino Unido: Eudema. Durán, A. (2009). Educcación Inclusiva. Madrid: SICA.

Durán, À. (2009). La Educación Inclusiva. En À. Durán, La Educación Inclusiva (pág. 152). España: SICA. Jean, P. (18 de Abril de 2013). El conocimiento. Recuperado el 09 de Noviembre de 2014, de El conocimiento: http://karenpallarescardoza/ jeanpiaget

Alcantara. (1992). Reseña del libro "Motivación". En P. P. Isabel, Motivación (pág. 280). España: Graphoprin.

Conferencia Mundial en Jomtien. (2000). Recuperado el Agosto de 2013, de Conferencia Mundial sobre Educación para todos y todas http//www.educ.para todos. 